\title{
THE COHOMOLOGY OF RESTRICTED LIE ALGEBRAS AND OF HOPF ALGEBRAS ${ }^{1,2}$
}

BY J. PETER MAY

Communicated by W. S. Massey, November 6, 1964

Introduction. In theory, the bar construction suffices to calculate the homology groups of an augmented algebra. In practice, the bar construction is generally too large (has too many generators) to allow computation of higher-dimensional homology groups. In this paper, we outline a procedure which simplifies the calculation of the homology and cohomology of Hopf algebras.

Let $A$ be a (graded) Hopf algebra over a field $K$ of characteristic $p$. Filter $A$ by $F_{q} A=A$ if $q \geqq 0$ and $F_{q} A=(I(A))^{-q}$ if $q<0$, where $I(A)$ is the augmentation ideal. The associated graded algebra $E^{0} A, E_{a, r}^{0} A$ $=\left(F_{q} A / F_{q-1} A\right)_{q+r}$, is clearly a primitively generated (bigraded) Hopf algebra over $K$. By a theorem due to Milnor and Moore [4], this implies that $E^{0} A$ is isomorphic to the universal enveloping algebra of its restricted Lie algebra of primitive elements if $p>0$, and to the universal enveloping algebra of its Lie algebra of primitive elements if $p=0$.

Our procedure is to calculate $H^{*}(A)=\operatorname{Ext}_{A}(K, K)$ by means of a spectral sequence passing from $H^{*}\left(E^{0} A\right)$ to $H^{*}(A)$. The fundamental result is the construction of a reasonably small canonical $V(L)$-free resolution of the ground field, where $V(L)$ is the universal enveloping algebra of a restricted Lie algebra $L$. We also obtain such a $U(L)$ free resolution, where $U(L)$ is the universal enveloping algebra of a Lie algebra $L$. These resolutions allow computation of the $E_{2}$ term of the cited spectral sequence.

The author would like to express his deep gratitude to J. C. Moore, who suggested this approach to the problem of calculating the cohomology of Hopf algebras.

STATEMENT OF RESUlTs. We first state the existence theorem for the required spectral sequence. Let $A$ be a filtered augmented graded algebra over a field $K$. Let $M$ be a left $A$-module and filter $M$ by $F_{q} M=\left(F_{q} A\right) M$. Then $E^{0} M$ is a left $E^{0} A$-module. Suppose that for $N=A$ and $N=M$ we have $N=\lim \operatorname{inv} N / F_{q} N$ and $N$ is of finite type as

\footnotetext{
${ }^{1}$ During the preparation of this paper, the author was partially supported by National Science Foundation grant number NSF-GP-1853.

2 The work announced here is contained in the author's doctoral thesis, submitted to Princeton University.
} 
a $K$-module. Let $M^{*}$ denote $\operatorname{Hom}_{K}(M, K)$, the right $A$-module dual to $M$. Under these hypotheses, we have

THEOREM 1. There exists a spectral sequence $\left\{E_{r} M^{*}\right\}$ satisfying

(i) $E_{2} M^{*}=\operatorname{Ext}_{E^{0} A}\left(K,\left(E^{0} M\right)^{*}\right)$.

(ii) Each $E_{r} M^{*}$ is a right differential $E_{r} K$-module, the module structure on $E_{2} M^{*}$ being given by the Yoneda product.

(iii) $E_{\infty} M^{*}$ is isomorphic to $E^{0} \operatorname{Ext}_{A}\left(K, M^{*}\right)$ (with respect to a suitable filtration) as a right $E^{0} H^{*}(A)$-module.

The spectral sequence $\left\{E_{r} M^{*}\right\}$ is obtained as the dual of a spectral sequence $\left\{E^{r} M\right\}$ passing from $\operatorname{Tor}^{A^{0} E}\left(K, E^{0} M\right)$ to $E^{0} \operatorname{Tor}^{A}(K, M)$. The latter spectral sequence is constructed by filtering $B(A) \otimes_{A} M$ in such a manner that $E^{0}\left(B(A) \otimes_{A} M\right) \cong B\left(E^{0} A\right) \otimes_{E^{0} A} E^{0} M$ as a $K$ module, $d_{0}=0$, and $E^{1}\left(B(A) \otimes_{A} M\right) \cong B\left(E^{0} A\right) \otimes_{E^{0} A} E^{0} M$ as a complex. (Here $B(A)=\bar{B}(A) \otimes A$ is the right bar construction.) The condition $N=\lim$ inv $N / F_{q} N, N=A$ and $N=M$, is needed to ensure the convergence of the spectral sequences (see [1]).

In order to calculate the differentials in the homology spectral sequence, we must know representative cycles in $B\left(E^{0} A\right) \otimes_{E^{0} A} E^{0} M$ for elements of $\operatorname{Tor}^{E^{0} A}\left(K, E^{0} M\right)$. However, we envision calculation of $\operatorname{Tor}^{E^{0} A}\left(K, E^{0} M\right)$ by means of an $E^{0} A$-free resolution of $K$ other than $B\left(E^{0} A\right)$. The following proposition will give an embedding of this resolution in $B\left(E^{0} A\right)$.

Proposition 2. Let $A$ be an augmented algebra over a field $K$. Let $X=Y \otimes A$ be an $A$-free complex over $K$ satisfying

(i) $X_{0}=A$ and $X_{0} \rightarrow K$ is the augmentation of $K$, and

(ii) no element of $Y$ is a cycle of $X$.

Then there exists a unique monomorphism $f: X \rightarrow B(A)$ of $A$-complexes over $K$ such that $f_{0}: X_{0} \rightarrow B_{0}(A)$ is the identity map of $A$ and $f(Y) \subset \bar{B}(A)$. $f$ is defined by the inductive formula $f(y)=S f d(y), y \in Y$, where $S$ is the contracting homotopy of $B(A)$.

It remains to define the promised $V(L)$-free resolution of the ground field, where $V(L)$ is the universal enveloping algebra of a restricted Lie algebra $L$.

We suppose first that $L$ is a Lie algebra over a field $K$ and construct a $U(L)$-free resolution of $K$. Let $L^{+}$, respectively $L^{-}$, denote the subspace of $L$ of elements of even degree, respectively odd degree, and adopt the convention that $L^{+}=L$ and $L^{-}$is void if the characteristic of $K$ is two. Consider $L$ as bigraded with bidegree ("homological degree") zero, and let $s L$ denote a copy of the $K$-space $L$ in which all elements have bidegree one. As a $K$-space, our $U(L)$-free resolution 
will be $Y(L)=\Gamma\left(s L^{-}\right) \otimes E\left(s L^{+}\right) \otimes U(L)$, where $\Gamma$ denotes a divided polynomial algebra and $E$ an exterior algebra. Let $f=\gamma_{r_{1}}\left(x_{1}\right) \ldots$ $\gamma_{r_{m}}\left(x_{m}\right)$ and $g=\left\langle y_{1}, \cdots, y_{n}\right\rangle$ denote typical elements of $\Gamma\left(s L^{-}\right)$and $E\left(s L^{+}\right)$. Let $f_{i}$ result from $f$ by replacing $r_{i}$ by $r_{i}-1$, and let $f_{i, j}$ $=\left(f_{j}\right)_{i}, i \leqq j$. Let $g_{i}$ result from $g$ by omission of $y_{i}$, and let $g_{i, j}=\left(g_{j}\right)_{i}$, $i<j$. With this notation, we have

TheOREM 3. Let $L$ be a Lie algebra over a field $K$. Give $Y(L)$ a differential by

$$
d(f g u)=d(f g) u, \quad u \in U(L),
$$

and

$$
\begin{aligned}
d(f g)= & \sum_{i=1}^{n}(-1)^{i+1} f g_{i} y_{i}+(-1)^{n} \sum_{j=1}^{m}\left(f_{j} g x_{j}-\frac{1}{2} f_{j, j} g\left\langle\left[x_{j}, x_{j}\right]\right\rangle\right) \\
& +(-1)^{n+1}\left(\sum_{1 \leq i<j \leqslant n}(-1)^{i+j} f g_{i, j}\left\langle\left[y_{i} y_{j}\right]\right\rangle+\sum_{1 \leq i<j \leq m} f_{i, j} g\left\langle\left[x_{i}, x_{j}\right]\right\rangle\right) \\
& +\sum_{i=1}^{m} \sum_{j=1}^{n}(-1)^{j} f_{i} \gamma_{1}\left(\left[x_{i}, y_{j}\right]\right) g_{j} .
\end{aligned}
$$

Then $Y(L)$ is a $U(L)$-free resolution of $K$.

In the case where $L$ is a restricted Lie algebra, we wish to enlarge the $V(L)$-free complex $W(L)=\Gamma\left(s L^{+}\right) \otimes E\left(s L^{-}\right) \otimes V(L)$ to obtain a resolution. In order to do this, the following corollary is needed.

CoRollary 4. Give $Y(L)$ a structure of $K$-algebra by requiring the product to agree with the natural one on $\Gamma\left(s L^{+}\right) \otimes E\left(s L^{-}\right)$and on $U(L)$ and to satisfy the relations

$$
\begin{array}{rlrl}
\text { (i) } & y_{1}\left\langle y_{2}\right\rangle-\left\langle y_{2}\right\rangle y_{1} & =\left\langle\left[y_{1}, y_{2}\right]\right\rangle, \quad y_{1} \in L^{+}, \quad y_{2} \in L^{+}, \\
\text {(ii) } & x\langle y\rangle+\langle y\rangle x & =\gamma_{1}([y, x]), \quad y \in L^{+}, \quad x \in L^{-}, \\
& \text {(iii) } \quad y \gamma_{r}(x)-\gamma_{r}(x) y=\gamma_{r-1}(x) \gamma_{1}([y, x]), \quad y \in L^{+}, \quad x \in L^{-}, \\
\text {(iv) } & x_{1} \gamma_{r}\left(x_{2}\right)-\gamma_{r}\left(x_{2}\right) x_{1} & =-\gamma_{r-1}\left(x_{2}\right)\left\langle\left[x_{1}, x_{2}\right]\right\rangle, \quad x_{1} \in L^{-}, \quad x_{2} \in L^{-} .
\end{array}
$$

Then $Y(L)$ is a differential $K$-algebra.

Now we suppose that the characteristic of $K$ is $p>0$ and that $L$ is a restricted Lie algebra with restriction ( $p$ th power) $\xi: L^{+} \rightarrow L^{+}$. $W(L)$ is a $V(L)$-free complex and a differential $K$-algebra, but is not exact since $\langle y\rangle y^{p-1}-\langle\xi(y)\rangle$ is a nonbounding cycle, $y \in L^{+}$. Let $s^{2} \pi L^{+}$ denote a copy of $L^{+}$with the degree of each element multiplied by $p$ and with each element having bidegree two. Let $\left\{y_{i}\right\}_{i \in I}$ be a basis for $L^{+}$indexed on a totally ordered set $I$. Identify $\Gamma\left(s^{2} \pi L^{+}\right)$as a $K$ - 
space with $\bigotimes_{i \in I} \Gamma\left(\tilde{y}_{i}\right), \tilde{y}_{i}=s^{2} \pi y_{i}$, where $\Gamma\left(\tilde{y}_{i}\right)$ precedes $\Gamma\left(\tilde{y}_{j}\right)$ in the tensor product if $i<j$. As a $K$-space, our $V(L)$-free resolution of $K$ will be $X(L)=\Gamma\left(s^{2} \pi L^{+}\right) \otimes W(L)$, and we will have $d\left(\gamma_{1}\left(\tilde{y}_{i}\right)\right)=\left\langle y_{i}\right\rangle y_{i}^{p-1}$ $-\left\langle\xi\left(y_{i}\right)\right\rangle$. Obviously $L^{+}$is a subrestricted Lie algebra of $L$ and therefore $W\left(L^{+}\right)$is defined. Let $Q\left(y_{i}\right)$ be the truncated polynomial algebra $P\left(y_{i}\right) /\left(y_{i}^{p}\right)$ and identify $V\left(L^{+}\right)$as a $K$-space with $\otimes_{i \in I} Q\left(y_{i}\right)$, where $Q\left(y_{i}\right)$ precedes $Q\left(y_{j}\right)$ if $i<j$ (this is possible by the Poincaré-BirkhoffWitt theorem). Then $X(L)$ may be given a structure of left $W\left(L^{+}\right)$module by means of the natural left $W\left(L^{+}\right)$-module structure on $W(L)$ and by

(a) $\left\langle y_{j}\right\rangle \gamma_{r}\left(\tilde{y}_{i}\right)=\gamma_{r}\left(\tilde{y}_{i}\right)\left\langle y_{j}\right\rangle$,

(b) $y_{j} \gamma_{r}\left(\tilde{y}_{i}\right)=\gamma_{r}\left(\tilde{y}_{i}\right) y_{j}+\gamma_{r-1}\left(\tilde{y}_{i}\right) \sum_{k=1}^{p-1}(-1)^{k}\left\langle y_{i},\left(\operatorname{ad} y_{i}\right)^{k}\left(y_{j}\right)\right\rangle_{i}^{p-1-k}$, $\left(\operatorname{ad} y_{i}\right) y_{j}=\left[y_{i}, y_{j}\right]$, and, inductively

(c) $y z \gamma_{r}\left(\tilde{y}_{i}\right)=y\left(z \gamma_{r}\left(\tilde{y}_{i}\right)\right)$, where $y z$ is an element of the basis for $V\left(L^{+}\right)$ defined above

With these definitions and notations, we can state

Theorem 5. Let $L$ be a restricted Lie algebra over a field $K$. Define a differential on $X(L)$ by

$$
d\left(\gamma_{r}\left(\tilde{y}_{i}\right)\right)=\gamma_{r-1}\left(\tilde{y}_{i}\right)\left(\left\langle y_{i}\right\rangle y_{i}^{p-1}-\left\langle\xi\left(y_{i}\right)\right\rangle\right),
$$

$$
\begin{aligned}
& \text { (ii) } \quad d\left(\gamma_{r_{1}}\left(\tilde{y}_{i_{1}}\right) \cdots \gamma_{r_{n}}\left(\tilde{y}_{i_{n}}\right)\right)=\sum_{j=1}^{n} \gamma_{r_{1}}\left(\tilde{y}_{i_{1}}\right) \cdots d\left(\gamma_{r_{j}}\left(\tilde{y}_{i_{j}}\right)\right) \cdots \gamma_{r_{n}}\left(\tilde{y}_{i_{n}}\right), \\
& \text { where } i_{k}<i_{l} \text { if } k<l \text {, and } \\
& \text { (iii) } d(g w)=d(g) w+g d(w), \quad g \in \Gamma\left(s^{2} \pi L^{+}\right), \quad w \in W(L) .
\end{aligned}
$$

Here the right sides of (ii) and (iii) are to be determined as elements of the $V(L)$-module $X(L)$ by means of the left $W\left(L^{+}\right)$-module structure of $X(L)$ and the algebra structure of $W(L)$. Then $X(L)$ is a $V(L)$ free resolution of $K$.

REMARKs. The difficult part of the theorem is the proof that $X(L)$ is actually a complex. The stated left $W\left(L^{+}\right)$-module structure is required to prove this. We make no attempt to give an explicit formula for the differential on $X(L)$, as its form would be quite complicated in the general case. The differential depends on the choice of the ordering of the set $I$ : if we interchange the order of two basis elements, the formula for the differential is changed. 
Finally, let $Z(L)=\epsilon_{i \epsilon I} \Gamma\left(\tilde{y}_{i}\right) \otimes W(L) \subset X(L)$ if $p>2$ and let $Z(L)$ $=X(L)$ if $p=2$. We define a diagonal map in $Z(L)$.

Theorem 6. Define $D: Z(L) \rightarrow Z(L) \otimes Z(L)$ by

$D$ is a morphism of $V(L)$-modules,

$$
\begin{aligned}
& D(\langle y\rangle)=\langle y\rangle \otimes 1+1 \otimes\langle y\rangle, \quad y \in L^{+}, \\
& D\left(\gamma_{r}(x)\right)= \sum_{i+j=r} \gamma_{i}(x) \otimes \gamma_{j}(x), \quad x \in L^{-}, \\
& D\left(\gamma_{r}(\tilde{y})\right)= \sum_{i+j=r} \gamma_{i}(\tilde{y}) \otimes \gamma_{j}(\tilde{y}) \\
&+\sum_{k=1}^{p-1} \sum_{i+j=r-1}(-1)^{k} \gamma_{i}(\tilde{y})\langle y\rangle y^{k-1} \otimes \gamma_{j}(\tilde{y})\langle y\rangle y^{p-1-k}, \\
& y \in\left\{y_{i}\right\}_{i \in I},
\end{aligned}
$$

(iii) $D(a b)=D(a) D(b)$, where the product on the right is to be determined by use of the algebra structure of $W(L)$ and, if $p=2$, by the left $W(L)$-module structure of $Z(L)$.

Then $D d=(d \otimes 1+1 \otimes d)$ on $Z(L)$.

REMARKs. In the case $p>2$, formula (iii) would not give a structure of differential co-algebra on all of $X(L)$, but $D$ may be extended to $X(L)$ by the use of Proposition 2 and the diagonal map of $B(V(L))$ (even though $D f X(L) \nsubseteq f X(L) \otimes f X(L)$ ). $D$ is co-associative only if $p=2$, although the resulting product on $H^{*}(V(L))$ is always associative.

Details and proofs of these results will appear elsewhere.

APPENDIX. We note here a curious corollary of Theorem 4. Let $L$ be an ungraded finite-dimensional restricted Lie algebra over a field $K$. Then we have:

Corollary 6. $\operatorname{Ext}_{V(L)}^{2}(K, K)=0$ implies that $L$ is Abelian and the pth power mapping $L \rightarrow L^{p}$ is one-to-one.

Proof. Consider the resolution $X(L)$ of Theorem 4 . $\operatorname{Ext}_{V(L)}^{2}(K, K)$ $=0$ implies $\operatorname{Tor}_{2}^{V(L)}(K, K)=0$, and therefore there are no nonbounding two-cycles in $\bar{X}(L)=X(L) \otimes_{V(L)} K$. No $\gamma_{1}(\tilde{x})$ is a summand of a boundary and $d \gamma_{1}(\bar{x})=-\langle\xi(x)\rangle$ in $\bar{X}(L)$. Thus $\xi(x) \neq 0$ and $L \rightarrow L^{p}$ is one-to-one. Further, if $\sum k_{i}\left\langle\xi\left(x_{i}\right)\right\rangle+\sum l_{j}\left\langle\left[y_{j}, z_{j}\right]\right\rangle=0, k_{i} \in K, l_{j} \in K$, and the $x_{i}$ are linearly independent, then $\sum k_{i} \gamma_{1}\left(\bar{x}_{i}\right)+\sum l_{j}\left\langle y_{j}, z_{j}\right\rangle$ is a nonbounding cycle unless each $k_{i}=0$. In particular, taking each $l_{j}=0$, if $\left\{x_{i}\right\}$ is a basis for $L$, then so is $\left\{\xi\left(x_{i}\right)\right\}$. But this implies $L \cap[L, L]$ $=\{0\}$, hence $L$ is Abelian.

Remarks. By results of Hochschild [2] and Jacobson [3], $[L, L]$ 
$=0$ and $K L^{p}=L$ if and only if every finite-dimensional $L$-module is completely reducible. The latter implies that $\operatorname{Ext}_{V(L)}^{1}(M, N)=0$ for all $V(L)$-modules $M$ and $N$, finite dimensional or not, and therefore all $V(L)$-modules are projective and $V(L)$ has global dimension zero, i.e., $\operatorname{Ext}_{\nabla(L)}^{n}(M, N)=0$ for all $n \geq 1$ and for all $M$ and $N$.

\section{BIBLIOGRAPHY} 1-23.

1. S. Eilenberg and J. C. Moore, Limits and spectral sequences, Topology 1 (1961),

2. G. Hochschild, Representations of restricted Lie algebras of characteristic $p$, Proc. Amer. Math. Soc. 5 (1954), 603-605.

3. N. Jacobson, Lie algebras, Interscience, New York, 1962.

4. J. Milnor and J. C. Moore, On the structure of Hopf algebras, Ann. of Math. (to appear).

YALE UNIVERSITY

\section{THE COHOMOLOGY OF THE STEENROD ALGEBRA; STABLE HOMOTOPY GROUPS OF SPHERES ${ }^{1,2}$}

\section{BX J. PETER MAY}

Communicated by W. S. Massey, November 6, 1964

In this paper, we state some of the results obtained by application of the methods of [4] to the study of the cohomology of the Steenrod algebra $A$. In brief, our results are a complete determination of $H^{s, t}(A)$ for $t-s \leqq 42$ in the $\bmod 2$ case, and for $t-s$ $\leqq 2(p-1)\left(2 p^{2}+p+2\right)-4$ in the $\bmod p$ case, $p>2$. Due to the existence of the Adams spectral sequence [1], these results give information about the stable homotopy groups of spheres.

We recall that the $\bmod p$ Adams spectral sequence $\left\{E_{r}\right\}$ (for the sphere) has differentials $\delta_{r}: E_{r}^{s, t} \rightarrow E_{r}^{s+r, t+r-1}$ and satisfies the properties:

$$
E_{2}^{s, t} \cong H^{s, t}(A) \text { as a } Z_{p} \text {-algebra. }
$$

(3) $\left\{E_{\infty}^{s, t} \mid t-s=k\right\}$ provides a composition series for $\pi_{k}(S ; p)$ (relative to a suitable filtration); here $\pi_{k}(S, p)$ denotes the stable homotopy group $\pi_{k}(S)$ modulo the subgroup of elements whose order is finite and prime to $p$.

${ }^{1}$ During the preparation of this paper, the author was partially supported by National Science Foundation grant number NSF-GP-1853.

2 The work announced here is contained in the author's doctoral thesis, submitted to Princeton University. 